$\mathbb{T}$ periodica polytechnica

\author{
Transportation Engineering \\ $40 / 1(2012) 33+38$ \\ doi: 10.3311/pp.tr.2012-1.06 \\ web: http://www.pp.bme.hu/tr \\ (c) Periodica Polytechnica 2012
}

RESEARCH ARTICLE

\section{Vehicle test based validation of a tire brush model using an optical velocity sensor}

Bálint Szabó

Received 2012-09-27

\begin{abstract}
In this paper a vehicle model validation process is introduced. For low velocity motions multibody dynamical tire model was developed based on tire brush model. It is described by several parameters, which should be identified. The accuracy of the tire model depends on the success of this identification. The validation of a tire brush model is a hard task, therefore the vehicle model was validated instead of the tire. The trajectory of the vehicle model is determined by the implemented tire model, thus the validation of the vehicle model should validate the tire model partly. During the validation process different low speed manoeuvres were carried out. The steering angle, steering torque, the longitudinal and lateral speed of the vehicle was recorded during the measurements. A simulation environment was set up in which the same manoeuvres were performed and the outputs from the tests and from the simulations were compared.
\end{abstract}

\section{Keywords}

Tire model validation $\cdot$ low velocity simulation

Department of Automobiles, BME, 6 Stoczek street H-1111 Budapest, Hungary e-mail: balint.szabo@auto.bme.hu

\section{Introduction}

There are vehicle systems which have significant influence on the dynamics of the vehicle. Therefore, for the development of these systems it is essential to develop accurate vehicle and tire model to able to reproduce the vehicle's behaviour in a simulation environment [10,11]. The developed vehicle controller systems are analysed in computer simulation. To ensure that the results of the simulations are appropriate, the models should be accepted to be realistic. It is important because the vehicle model informs the system how the vehicle would react for various input signals. To have a mathematical model, the dynamical differential motion equations of the vehicle have to be set up which describe the vehicle's motion. For each system or analysed problem a specialised model can be created. There are models which can reproduce the total vehicle motion in all the six degrees of freedom, but these kinds of models are used mainly in vehicle simulator programs which are applied for developing any kind of vehicle dynamical system. However, in several cases the use of a simplified model is enough which is able to describe only some basic motions of the vehicle, like longitudinal motion, or simple planar motion [2,3].

The model described in this article is a special vehicle model, which is required to examine the vehicle motion during low speed cornering manoeuvres. In this case the vertical movements, the roll and the pitch of the body are neglected. The vehicle with wheels is represented in top view and its position is described by three parameters: two translational and one rotational coordinate. Usually a simple, two-wheeled bicycle model is developed to describe the vehicle's planar motion, but in this case a four wheeled vehicle model is used. A vehicle with four wheels is kinematical overdetermined, therefore it is hard to describe the motion of the vehicle. For this, appropriate wheel model is required which consists of the wheel rim and the tire.

The reasons why the road vehicles are equipped with pneumatic tires are the following: reducing the high frequency vibration delivered from the road to the vehicle, ensuring adhesion on the road and ensuring load distribution on uneven surface. These are the main properties which should be reproduced by an adequate tire model. The model should simulate the elasticity and 
the damping effect, the adhesion and the load distribution. In our model the elasticity has a significant role: it balances the kinematical overdetermined vehicle by the deformation of the tires.

The last step of the model development is a validation process. Since the model contains uncertainty due to the simplifications and due to the inaccurate parameter identification, it should be verified that the model behaves just like the real system. During the model validation, measurements are carried out on a real system, and simulations are performed using the same inputs. The results of the measurements and the simulations are compared to each other. Finally consequences should be drawn whether the model can be accepted for low speed manoeuvres or not.

\section{Tire model}

The tire model is based on the brush model [1]. The wheel rim is a rigid body, its degrees of freedom are reduced by geometrical constrains: only the longitudinal, lateral movements and the rotation about the spin and the steering axes are allowed. Cambering and the vertical motion are ignored. These simplifications can be made because only the planar motion of the vehicle is analysed. The tire carcass is discretised along its circumference and its total mass is distributed to point masses. These tire elements are connected to each other and to the wheel rim via spring-damper elements (Fig. 1a). These spring-damper elements represent the stiffness and the damping of the tire. This model would be appropriate to simulate all the possible deformations of the tire. Although, the radial deformation is neglected, therefore all the tire elements have two degrees of freedom: tangential and lateral displacements. During the planar motion, especially in case of low speed manoeuvres, there is no change in the radial deformation of the tire, therefore it can be ignored. However each pneumatic tire suffers static radial deformation when it is loaded vertically and this cannot be neglected, since it determines the contact patch of the tire. This static deformation can be taken into account in the model without releasing the radial deformation of the tire elements. An additional geometrical constraint can be prescribed for the radial displacements of the tire elements: the trajectory of the radial deformation has a flatted circle shape instead of a circular geometry (Fig. 1p). During the deformation of the tire the point masses can move on the surface of this deformed ring. On Fig. $1 \mathrm{p}$ only the tangential motion can be represented the lateral deformation of the elements are perpendicular to the centre plane of the wheel.

The flatted plane of the wheel is always in touch with the ground. The tire elements, located in this contact patch, are also in contact with the ground, therefore friction force acts on them.

When the wheel starts to move or rotate in any of its possible directions, deformation arises between the tire elements and the wheel rim. When the wheel is rotated about its spin axis, only the wheel-side endpoints of the springs start to move along

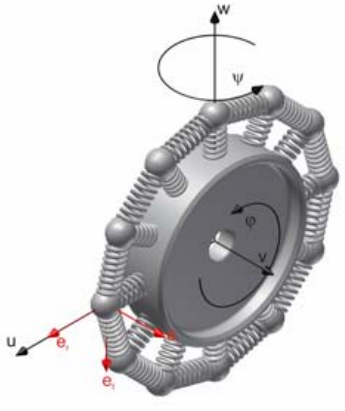

(a) Spatial structure

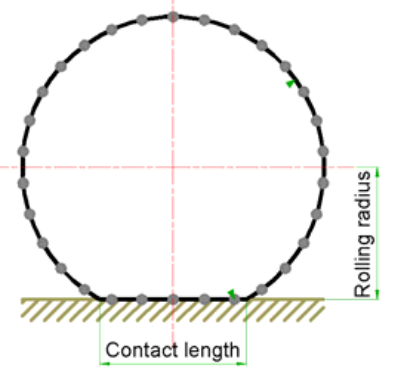

(b) Deformed shape
Fig. 1. Multibody dynamical tire brush model

the circumference of the wheel rim. Certainly the flatted cylinder does not rotate the flat surface of the wheel rim remains in contact with the ground [4, 6].

The dynamics of the wheel is described by Newton's second law. Before the mathematical model is set up, coordinate systems should be defined. There is a global coordinate system $\langle\xi, \eta\rangle$ which is grounded, and in which the rigid body motions, in this case the motion of the wheel rim can be described. There are two local coordinate systems: the $\langle u, v, w\rangle$ fixed to the wheel rim, and the $\left\langle e_{t}, e_{v}\right\rangle$ fixed to each tire element (Fig. 3a). The wheel disc has four degrees of freedom: longitudinal displacement $(u)$, lateral displacement $(v)$, spinning $(\phi)$ and steering $(\delta)$. It can be described by four second order differential equation, one for each global direction with

$$
\begin{aligned}
& m \ddot{\xi}=F_{t, \xi}+F_{r e s, \xi} \\
& m \ddot{\eta}=F_{t, \eta}+F_{r e s, \eta} \\
& J_{w} \ddot{\delta}=T_{t, w}+T_{s t} \\
& J_{v} \ddot{\phi}=T_{t, v}+T_{d r v}
\end{aligned}
$$

where $m$ is the mass of the wheel; $J_{v}$ and $J_{w}$ are the mass moments of inertia about the spin and steering axes; $F_{t, \xi}$ and $F_{t, \eta}$ are the components of the tire forces; $T_{t, w}$ and $T_{t, v}$ are the components of the tire torques; $F_{r e s, \xi}, F_{\text {res }, \eta}$ are the resistance forces like wind drag or road slope; $T_{s t}, T_{d r v}$ are the steering and the drive or brake torques.

Each tire element has two degrees of freedom: tangential displacement $\left(e_{t, i}\right)$ and the lateral deformation $\left(e_{l, i}\right)$ represented on Fig. 3a. The deformation of each tire element is determined by second order differential equations:

$$
\left\{\begin{array}{c}
m_{t} \ddot{e}_{t, i}=b_{t, t}\left(e_{t, i+1}-2 e_{t, i}+e_{t, i-1}\right)+ \\
d_{t, t}\left(\dot{e}_{t, i+1}-2 \dot{e}_{t, i}+\dot{e}_{t, i-1}\right)-b_{w, t} \Delta e_{t, i}-d_{w, t} \dot{e}_{t, i}+F_{i, f, t} \\
m_{t} \ddot{e}_{l, i}=b_{t, l}\left(e_{l, i+1}-2 e_{l, i}+e_{l, i-1}\right)+ \\
d_{t, l}\left(\dot{e}_{l, i+1}-2 \dot{e}_{l, i}+\dot{e}_{l, i-1}\right)-b_{w, l} \Delta e_{l, i}-d_{w, l} \dot{e}_{l, i}+F_{i, f, l}
\end{array}\right.
$$

Where $m_{t}$ is the mass of a single tire element; $b$ is the stiffness; $d$ is the damping; $F_{f, t}$ and $F_{f, l}$ are the friction force components. Regarding to the stiffness and damping the first index identifies the location $(t$ : between tire elements, $w$ between tire element 
and wheel rim); the second index shows the direction ( $t$ for tangential, $l$ for lateral).

\section{Vehicle tests}

To validate the tire and the vehicle model vehicular measurements were carried out. During the validation measurements and simulations are carried out with the same excitation and initial conditions. The results of the simulation and the results of the tests are compared. These results should be similar in an expected measure to be able to declare that the model behaves similarly to the real vehicle.

The best way to validate a tire model would be to measure the tire deformation and the tire forces during different vehicle manoeuvres [7]. Measuring the tire deformation on a moving vehicle is a very hard task [9], and for the wheel force measurement expensive devices are required [8]. Therefore we decided to validate the vehicle model into which the multibody dynamical tire model has been implemented. During the validation the vehicle's motion state, the vehicle's velocity was recorded.

For the tests a Chrysler Voyager was selected and it was equipped with the following measurement devices (Fig. 2). Into the steering system a steering torque and steering angle sensor were inserted and on the chassis of the vehicle an optical vehicle speed sensor was mounted. With the help of these equipments the steering angle, the steering torque, the longitudinal, the lateral and the absolute velocities can be recorded during measurements. Before the measurements some identification was performed, because the simulation model should have the same parameters as the test vehicle has. The geometrical parameters and the wheels loads can be measured easily. The tire stiffness in longitudinal and lateral direction was identified on a tire test bench. Further parameters of the steering system and the friction coefficient values were determined.

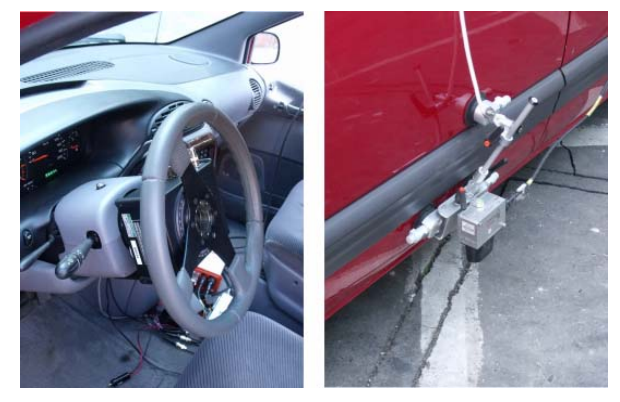

Fig. 2. Measurement system of the test vehicle

For the measurement of the steering angle characteristic, rotatable base with a scale division was placed under the front steered wheels. The scale on this rotatable base shows the current angle of the steered wheels meantime the angle of the steering wheel is recorded by the steering transducer system. The steering wheel was turned from the right side to the left side during angles of the steering wheel and the steered wheel were recorded. As it was expected, the real steering characteristic deviates from the theoretical Ackerman geometry at larger steering angles. The reason is that the suspension parameters are a result of a compromise, therefore at larger steering angles the deviation from the ideal characteristic is accepted, since this case the vehicle speed is low. (Fig. 3)

The damping of the steering system is measured the following way. The steering wheel was turned until the front tires started to slide. Then, the steering wheel was released, and the oscillation of steering system was recorded (Fig. 4). Using the method of the logarithmical decrement the relative damping was determined.

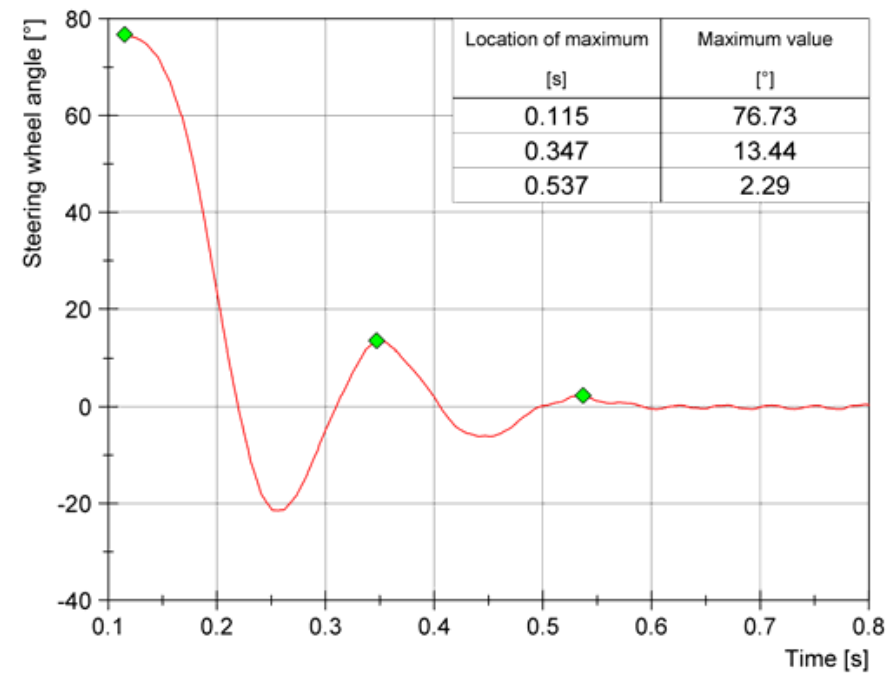

Fig. 4. Oscillation in steering angle

One more parameter had to be identified: the friction coefficient. On the test track, the steering wheel was turned from the middle position to the right side final position and from the right side to the left side final position. The engine was stopped in order to eliminate the effect of the hydraulic servo steering system that would modify the steering torque on the steering wheel. During the measurement, the steering torque and steering angle were recorded (Fig. 5). If we observe the initial part of this curve, then we can establish that it starts with a linear section. In this domain the tire is deforming only, thus the slope of the curve represents the stiffness of the front tires. After reaching a maximum value, the steering torque reduces, and sets in a lower, stable value. The maximum value belongs to the static friction, the steady state value belongs to the sliding friction.

Since the measured torque is related to the steering wheel, it has to be converted to one of the steered wheels. Furthermore the measured torque also contains some extra internal friction torques of the steering system. To determine these internal losses, the same steering manoeuvre was performed standing on rotatable base with the front wheels. In this case only the steering loss is measured, since neither friction force nor tire deformation arise. The recorded steering torque is reduced with this internal friction torques, and then the resulting torque covers the tire friction and the deformation forces. For the transformation of the measured steering torque to the steered wheels the recorded steering characteristic was used. However, the con- 

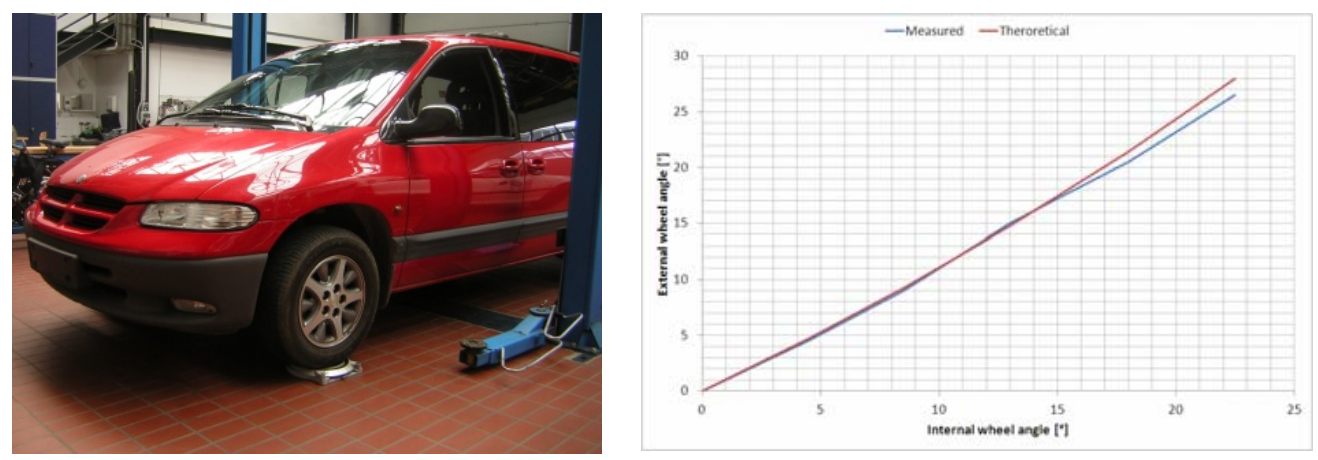

Fig. 3. Steering angle measurement and the characteristic
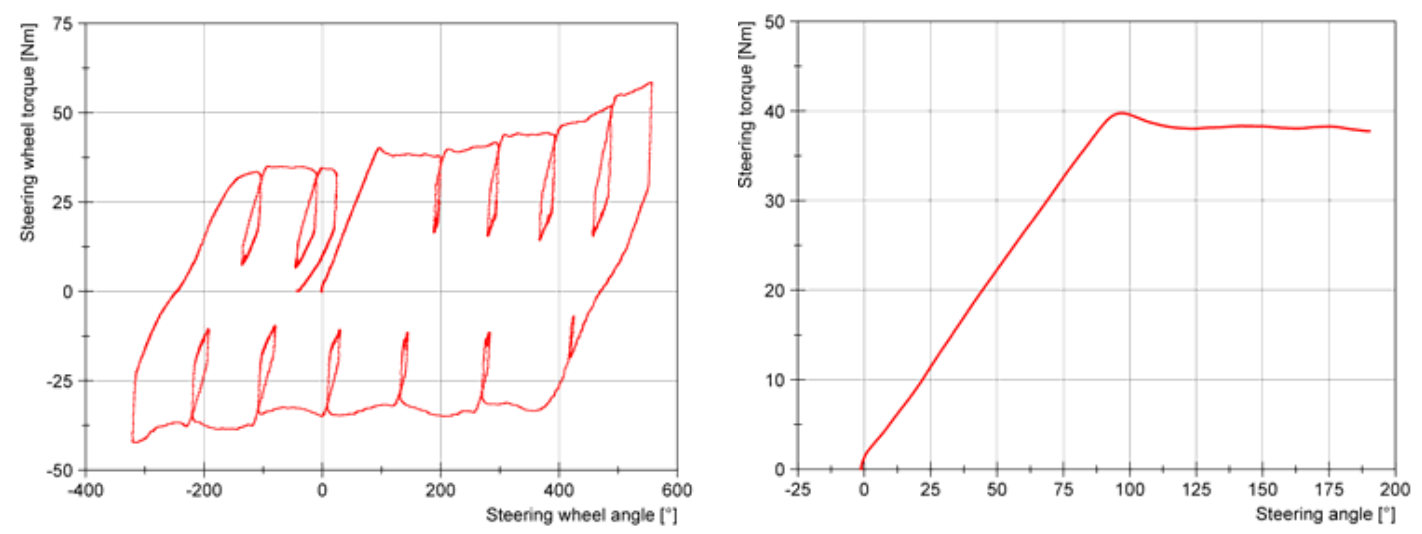

Fig. 5. Steering torque during steering at standstill, total (left) and zoomed (right) view

verted value is the total torque which is the sum of the torques arising on both steered wheels. Since the steering angle was low, it can be considered that the steered wheels rotation is similar, and this torque distributes between them in proportion of the wheel load. The resulting torque function covers the steering torque on one steered wheel. The steering torque causes linearly increasing distributed load in the contact area which can be substituted by concentrated force vector. From this force we can calculate the friction coefficient using the known wheel load.

As it was mentioned earlier different manoeuvres was performed during vehicle tests. All of them are low speed cornering manoeuvres. Three manoeuvres were defined with different steering functions. The test vehicle is equipped with automatic transmission, so during the test only the brake pedal was released, and the vehicle ran at its creeping velocity. The first test was a sinusoidal steering manoeuvre at constant speed (Fig. 6). The manoeuvre started with zero steering angle. After the vehicle had accelerated to a low speed, a sinusoidal steering movement was made, finally the vehicle was stopped. At the second test the steering angle was turned to the right final position meantime the car was standing, and then the vehicle started to run a quarter circle (Fig. 7). Finally, the third test is similar to the second one, but this case the vehicle had started before the steering wheel was turned to the right final position (Fig. 8). From the measurements, the steering angle and the absolute velocity were used as input for the simulations. The velocity of the vehicle was measured at the right rear door, but in the simulation we use the velocity of the centre of the gravity, therefore a conversion had to be made.
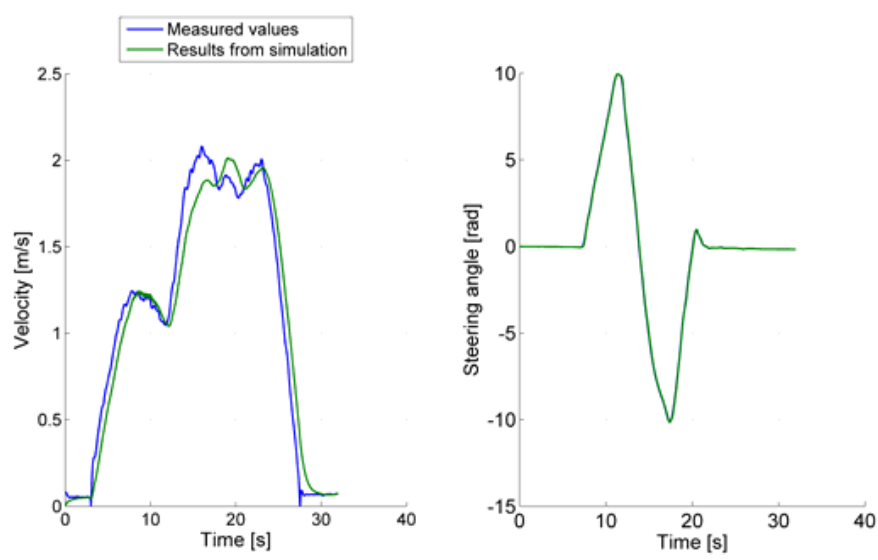

Fig. 6. Inputs for sinusoidal steering manoeuvre

A driver model was also implemented into the vehicle model to be able to reproduce the steering angle and the velocity. The driver model contains a simple $\mathrm{P}$ controller for the control of the absolute velocity. For the accurate reproduction, the steering controller was neglected, and the measured steering angle was used directly on the vehicle model.

During the first test manoeuvre the vehicle was accelerated to $2 \mathrm{~m} / \mathrm{s}$. When the steering manoeuvre started the velocity of the vehicle reduced a bit, but then the engine controller compensated the brake effect of the steering. The longitudinal velocity has similar shape to the absolute velocity, although the longitudinal velocity is with one order larger than the lateral speed. The lateral velocity function has a sinusoidal shape resulted by the 

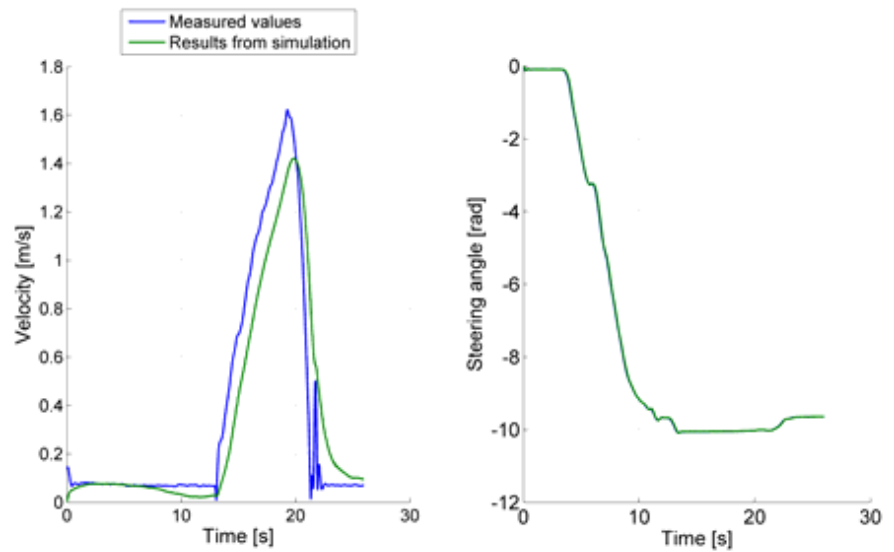

Fig. 7. Inputs for presteering manoeuvre

steering angle function (Fig. 9). The second was the presteering test, when the steering was done before vehicle starts. Both the longitudinal and the lateral velocity have the same shape, and they are also in phase. The only difference can be observed in the magnitudes. In this case the steering angle was constant during vehicle motion so the lateral velocity depends on the absolute velocity of the vehicle. As in the earlier case, the magnitude of the lateral velocity is lower than the magnitude of the longitudinal speed (Fig. 10). At the third manoeuvre, the steering wheel was turned after the vehicle starts. The shape of the velocity functions are the same, as it was in the case of the second manoeuvre (Fig. 11).
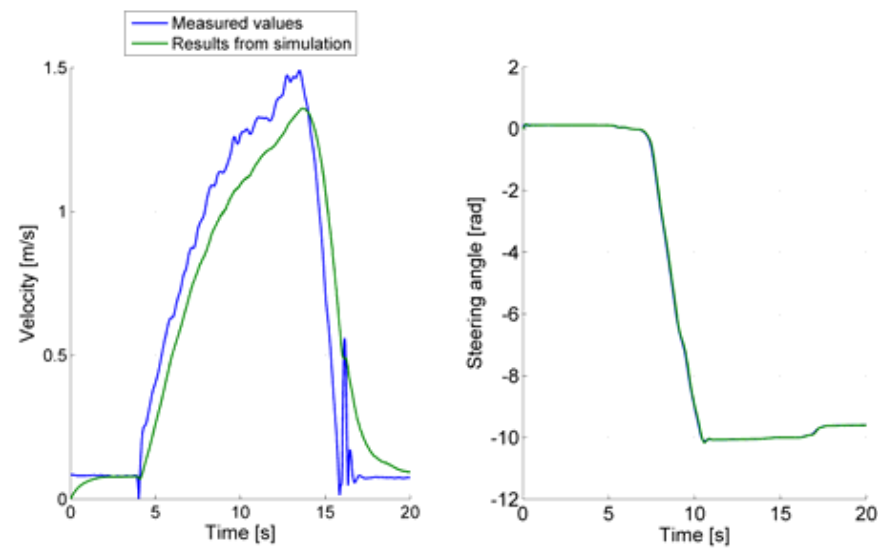

Fig. 8. Inputs for presteering manoeuvre

After comparing the simulation results with the measurements, it can be stated that in both cases we get similar results to the real vehicle test. Unfortunately these results are not enough to claim that the model is totally valid although the model can represent the real vehicle motions for low speed vehicle manoeuvres.

\section{Summary}

The purpose of my work was to examine whether the constructed tire model, implemented in a vehicle model, behaves in a realistic way, and the simulations can be used for low speed vehicle manoeuvres. Vehicle tests were performed for validation process. First, parameter identification tests were carried
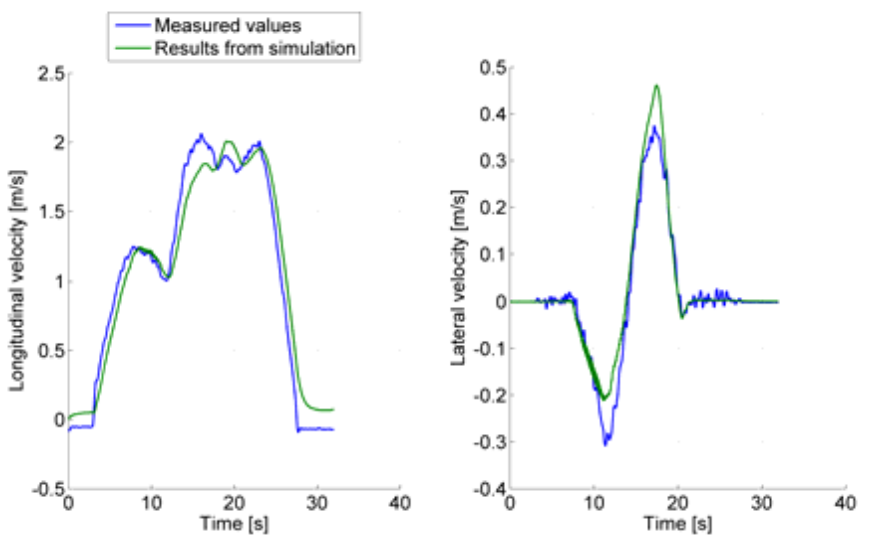

Fig. 9. Results of sinusoidal steering manoeuvre
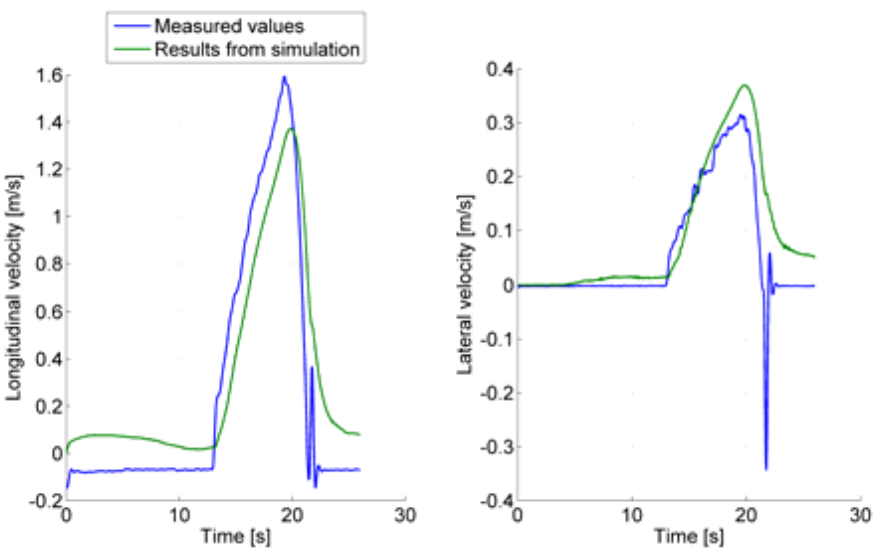

Fig. 10. Results of presteering manoeuvre
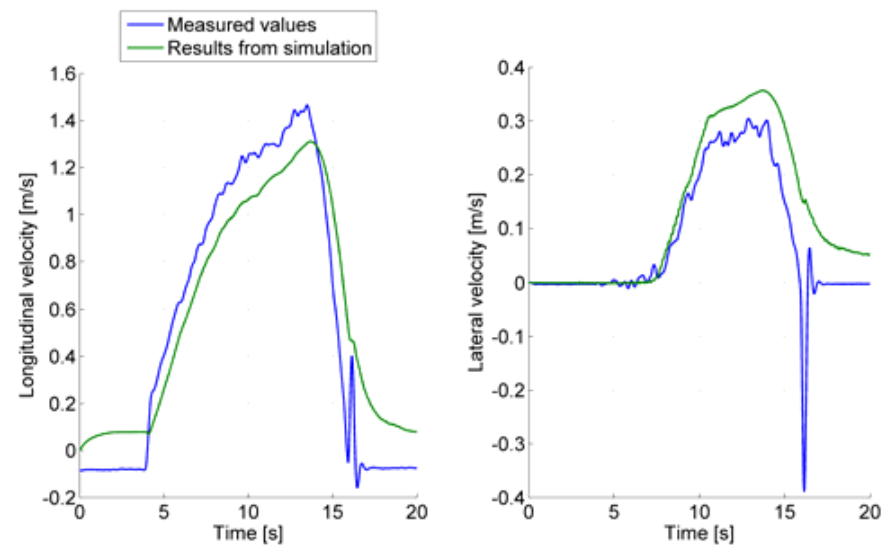

Fig. 11. Results of poststeering manoeuvre 
out to determine some geometrical and mass related parameters, the steering characteristic and the friction coefficient. Finally three vehicle manoeuvres were performed to compare the resulting motion state of the real vehicle to the simulated one. It was stated the results are similar, therefore the vehicle model calculates the vehicle motion properly, but it is valid only for the low speed motions.

\section{References}

1 Pacejka H B, Tyre and Vehicle Dynamics, Elsevier ButterworthHeinemann, 2002.

2 Lugner P, Plöchl M, Tyre Model performance test: First experiences and results, Vehicle System Dynamics 43 ( 2005), no. 1, 48-62.

3 Deur J, Asgari J, Hrovat D, A 3D Brush-type Dynamic Tire Friction Model, Vehicle System Dynamics 42 (2004), no. 3, 133-173.

4 Szabo B, Takacs D, Stepan G, Vehicle Model for an Automatizated Parking Control System, 2006. VSDIA Conference Budapest.

5 Szabo B, Takacs D, Vehicle Model Design and Vehicle Motion Analysis for an Automatizated Parking Manoeuvre, Budapest, 2008. Gepeszet Conference.

6 Szabo B, Palkovics L, Analysis of Low Speed Steering Manoeuvre with Dynamical Tire Models, Stockholm, 2009. 21st International Symposium on Dynamics of Vehicles on Roads and Tracks.

7 Driveability Testing Alliance, Driveability Test Maneuver: Steady-state Circular Test, 2009. Test Specification.

8 Klaus W, Barz D, An Example for Camber and Wheel Force Measurements for the Generation of Test Stand Data and Validation Purposes, 2010, available at www. corrsys-datron. com Technical Specification.

9 Wehrhahn D, Non-contact laser measurement in the tire and rubber industry. Measuring Systems for Quality Assurance.

10 Hankovszky Z, Kovacs R, Palkovics L, Electronic stability program with vehicle sideslip estimation, Periodica Polytechnica.

11 Bóka G, Márialigeti J, Lovas L, Trencseni B, Face dog clutch engagement at low mismatch speed, Periodica Polytechnica Ser. Tr. Eng. ( 2010), 29-35. doi=10.3311/pp.tr.2010-1.06 